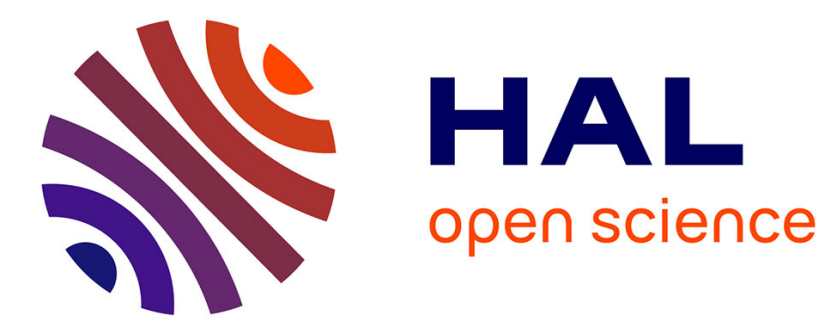

\title{
Ultra stable high power laser for the VIRGO project C.N. Man
}

\section{To cite this version:}

C.N. Man. Ultra stable high power laser for the VIRGO project. International Conference: Laser M2P 3, 1993, Lyon, France. pp.C4-311-C4-319, 10.1051/jp4:1994476 . jpa-00252739

\section{HAL Id: jpa-00252739 https://hal.science/jpa-00252739}

Submitted on 1 Jan 1994

HAL is a multi-disciplinary open access archive for the deposit and dissemination of scientific research documents, whether they are published or not. The documents may come from teaching and research institutions in France or abroad, or from public or private research centers.
L'archive ouverte pluridisciplinaire HAL, est destinée au dépôt et à la diffusion de documents scientifiques de niveau recherche, publiés ou non, émanant des établissements d'enseignement et de recherche français ou étrangers, des laboratoires publics ou privés. 


\title{
Ultra stable high power laser for the VIRGO project
}

\author{
C.N. MAN
}

Virgo-LAL, Bât. 208, Campus d'Orsay, 91405 Orsay cedex, France

\section{Introduction}

VIRGO is a French-Italian project for the interferometric detection of gravitational waves. It is a large scale $\mathrm{km}$ antenna based on the Michelson interferometer with delay lines in each arm.

Gravitational waves are predicted by general relativity theory and by all other relativistic theories of gravity, and all the theories agree, in rough order or magnitude, on the strengths of the waves to be expected from astrophysical sources. Although gravitational waves have not yet been observed directly, the effect of the back-action of gravitational waves emission on one source which is the orbital decay of the binary pulsar $1013+15$ [1], has been measured and agrees with general relativity's predictions to within the experimental error of several percent.

The basic and first idea of the interferometric detection of GW resides in a paper of Gertsenshtein and Pustovoit [2], but the first complete work on the noises competing with the GW signal in an interferometric antenna is due to Weiss [3]; it is also his merit the idea of using a "stable" cavity such as the Herriot delay line, and fast light phase modulation for getting rid of the laser's amplitude fluctuations. But the very first experimental attempt, giving high sensitivity in the test masses displacement measurement, is due to Forward [4], who used retroreflectors to reflect the beam back to a beam splitter and used active controls for locking the interferometer to a fringe; he obtained a spectral strain sensitivity of $\tilde{h}>2.10^{-16} \mathrm{~Hz}^{-1 / 2}$ for frequencies above $2 \mathrm{kHz}$.

\section{Gravitational Wave effects}

Just as an electromagnetic wave pushes a charged particle that initially is at rest back and forth in a direction transverse to the wave's propagation, so also does a gravitational wave. If a test particle at location $(x, y, z)$ is unsconstrained by other forces, it will accelerate by an amount $\delta \dot{\vec{x}}=\vec{F} / m$ in response to the gravitational wave, and its resulting displacement will be: $\delta \tilde{x}=\frac{1}{2} \tilde{h} x, \delta \tilde{y}=-\frac{1}{2} \tilde{h} x, \delta \tilde{z}=0$. Note that the 
gravitational force fields are quadrupolar and are transverse to the wave's propagation direction. Since the displacement is proportional to the separation of the particle from the origin with proportionality factor $\hat{h}$, one can regard it as strains of space and a typical order of magnitude is $10^{-22} \mathrm{~Hz}^{-1 / 2}$ around the frequency of $1 \mathrm{kHz}$.

So our sensitivity goal for the Virgo antenna is the above strain sensitivity and we start our laser specification requirements from that order of magnitude.

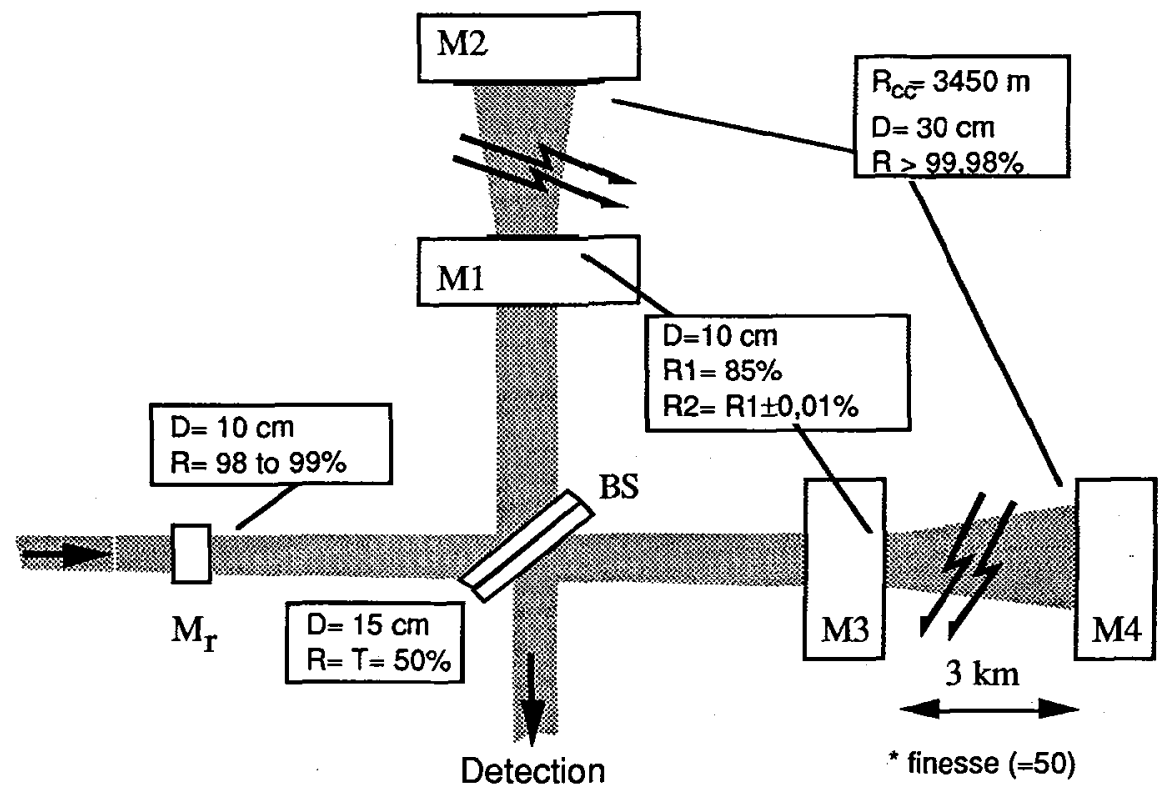

Figure 1: Schematic representation of a recycling Michelson interferometer

Figure 1 is a simplified schematic diagram of an interferometric detector of the type of Virgo. We shall denote by $L \sim L_{x} \sim L_{y}$ the mean length of the nearly equal arms of the Michelson. If a gravitational wave propagates vertically and has its polarization axes parallel to the detector's arms, then the wave will move the end masses back and forth relative to the central mass. The resulting wave-induced changes in the $x$-arm and $y$-arm lengths will be $\delta \widetilde{L_{x}}=\frac{1}{2} \widetilde{h} L$ and $\delta \widetilde{L_{y}}=-\frac{1}{2} \widetilde{h} L$, i.e. they will be equal and opposite. These changes will be monitored by laser interferometry: laser beam sent from the central 
mass down to the arms and reflected off mirrors on the end masses, will return to the central mass with a relative phase change $\Delta \Phi$ that is proportional to the difference in the arms lengths, $\Delta \Phi=2 \pi \Delta L / \lambda$, where $\delta \tilde{L}_{x}-\delta \tilde{L}_{y}=\tilde{h}(t) L$.

The VIRGO interferometer is basically a Michelson interferometer (MITF), with two $3 \mathrm{~km}$ long, perpendicular arms. The large length of $3 \mathrm{~km}$ is chosen mainly to decrease the influence of displacement noises, like seismic noise and thermal noises. Each arm contains a "gravito-optic transducer", formed by a $3 \mathrm{~km}$ long Fabry-Perot cavity having a finesse of the order of 50 . This optimizes the sensitivity to gravitational waves in the frequency domain around $1 \mathrm{kHz}$, and prevents any difficulty with the radiation pressure effects due to the high power impinging on the cavity mirrors.

\section{Scientific motivations}

The expected scientific benefits of the detection of gravitational wave signals concern : 1) fundamental physics, 2) astrophysics, 3) applied physics. Moreover, 4) the Virgo experiment serves the useful role of "driver" in several fields of physics.

The obtainment of the aimed sensitivity necessitates to push to extreme limits existent technologies. The results obtained so far by the members of the Virgo project are already considerable. One can mention for instance :

- coherent addition of lasers [5],

- "recycling" of light [6],

- external modulation [7],

- ultrastabilization of a YAG laser $\left(10^{-3} \mathrm{~Hz}\right)$ [8],

- construction of a low-frequency seismic super-attenuator [9].

Let us note in passing that the Virgo experiment, through its need of very advanced technologies, can provide a stimulus for the industries of the countries involved in it.

It is also important to mention that besides the direct scientific benefits that are likely to come out of the Virgo experiment, this experiment has the useful effect of providing a motivation and/or a stimulus for several scientific researches. Let us quote :

- the theoretical and experimental studies of "squeezed states" (quantum optics),

- the development of new numerical methods (numerical relativity),

- the theoretical and experimental study of the nonlinear dynamics of a pendular Fabry-Perot (retarded differential equations) [10]. 


\section{Noise sources and sensitivity}

Sources of noise that can limit the sensitivity of an interferometric gravitational wave detector are of three types. First, there are random processes intrinsic to the measurement: shot noise of the light, quantum noise associated with the uncertainty principle, and thermal fluctuations in masses, mirrors, suspension wires and other physical elements, that induce motions of the optical surfaces. A second source is noise introduced by the environment; examples include the transmission of random seismic motions to the optical components. A third group of noise sources are technical: these are nonfundamental effects, those whose influence can be reduced by care and advances in the technology. Examples are interferometer phase fluctuations resulting from instabilitity of laser beam geometry, phase fluctuations from residual gas along the transmission paths, and scattering of light by mirrors and tube walls.

We will identify and estimate here only the noise sources coming from the laser as they influence the strain measurement. I will refer the reader to the Virgo proposal (1989) for the complete list of noise sources.

1. Photon shot noise: the ability to measure the optical phase difference at the output of the interferometer is limited by the quantum fluctuations of the light. The equivalent

gravitational-wave strain noise is: $\quad \tilde{h}_{s n}=\frac{\lambda}{2 \pi L} \sqrt{\frac{h v}{\eta P}}=2.10^{-23} \sqrt{\frac{1 k W}{\eta P}} \quad \mathrm{~Hz}^{-1 / 2}$ around $1 \mathrm{kHz}$ the expected sensitivity of $\tilde{h}=2.10^{-23} \mathrm{~Hz}^{-1 / 2}$ can be reached with an optimum length of $130 \mathrm{~km}$, obtained in Virgo by folding the $3 \mathrm{~km}$ arms about 40 times, and illuminating the interferometer with a $1 \mathrm{~kW}$ power incident on the central mass. The solution proposed in Virgo is the use of recycling technique consisting of including the whole interferometer inside a resonant cavity by adding a mirror $\mathbf{M}_{\mathbf{R}}$ (figure 1); the light incident on the Michelson is then multiplied by the storage factor of the cavity formed by this mirror and the whole Michelson sitting on the white fringe at the reflection port. This storage can be estimated to be more than 50 , putting the laser power constraints to $20 \mathrm{~W}$.

To get the $20 \mathrm{~W}$ stabilized laser required for Virgo, we propose the solution of injection locking a high power oscillator with a stable low power oscillator: stabilizing a high power oscillator requires intravity elements to ensure single frequency operation. These intracavity elements always introduce losses in the high power oscillator and thereby reduce its efficiency and output power. Furthermore, the frequency noise level required for Virgo demands complex active control to reduce the free-running linewidth of the high power Nd:YAG laser. For these reasons intracavity and active stabilization of a high power oscillator is an unattractive alternative. 


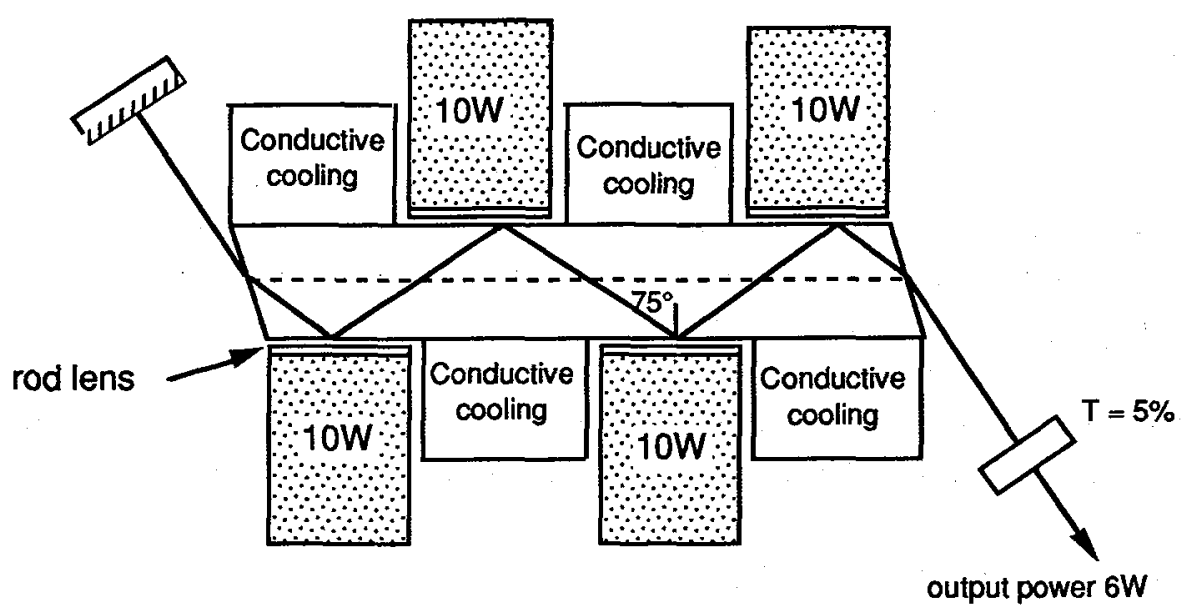

Figure 2: Diode-pumped Nd:YAG slab laser

The high power laser is a $\mathrm{cw}$ Nd: YAG slab laser transversely pumped by four high-power laser-diode (SDL 3490-S) as shown on figure 2. The slab is designed to have four total internal reflections in a single path. The angle of incidence at the reflections is chosen to be high $\left(75^{\circ}\right)$ so that the beam stays longer near the pumped surfaces where the gain is higher. The light of the diodes is collimated by using a rod lens ( $\phi=2 \mathrm{~mm}$ ). The size of the pump in the vertical direction ( $1 / \mathrm{e}^{2}$ beam diameter), measured with a scanning slit, about $800 \mu \mathrm{m}$, is variable by $+/-20 \%$ by changing the distance between the diode and the lens. In order to use the pump light which is not absorbed on the first pass, aluminium reflectors are placed on the other side of the slab. The total power absorbed in the slab is roughly $33 \mathrm{~W}$. In single transverse mode we obtain $6 \mathrm{~W}$ of polarized output power in a plano-plano cavity $450 \mathrm{~mm}$ in length. The optical-to-optical efficiency is $16 \%$. We are now in the process to scale up this laser to a power of $10 \mathrm{~W}$ and then use it for the first generation of Virgo.

2. Frequency fluctuations of the laser: the need for stabilization of the laser frequency arises from asymmetries between the two arms, in terms of storage time $\tau_{s}$ and. the existence of scattered light in the system. These two effects convert fluctuations of the laser frequency into phase shifts, and thus signals, at the output of the interferometer. The resulting gravitational wave strain noise is: $\tilde{h}_{f}=\frac{\Delta \tau_{s}}{\tau_{s}} \frac{\delta \tilde{v}(f)}{v_{0}}$ where $\delta \tilde{v}(f)$ is the 
spectrum of the laser frequency fluctuations, $\frac{\Delta \tau_{s}}{\tau_{s}}$ is the asymmetry between the arms and $v_{0}$ is the laser frequency. Assuming an asymmetry of $10^{-3}$, this corresponds to the frequency stability of $\delta v^{\tilde{\nu}}(f) \leq 10^{-6}(f / 1 \mathrm{kHz})^{2} \mathrm{~Hz} / \sqrt{\mathrm{Hz}}$. This stability is better than any existing oscillator today and will be achieved by a combination of active control and passive filtering, the former supplying stabilization at low frequencies, where large servo gains are possible, and the latter being effective at high frequencies, above the pole frequency of the optical filtering cavity formed by the recycling cavity that has a pole frequency around $5 \mathrm{~Hz}$. So the active control will be done in two stages: a prestabilization to a short rigid cavity, followed by a stabilization to the arms of the interferometer. The need for additional stabilization after the prestabilisation is twofold. First, there is the problem of getting enough loop gain in a single stage. The raw frequency noise of the source will be on the order of $10-100 \mathrm{~Hz} / \mathrm{JHz}$ from DC $-1 \mathrm{kHz}$. To reach a $\tilde{\delta} \tilde{v}(1 \mathrm{kHz})$ $=10^{-6} \mathrm{~Hz} / \sqrt{\mathrm{Hz}}$ for example, would require a loop gain of $10^{7}-10^{8}$ at $1 \mathrm{kHz}$, which is difficult given a loop unity gain frequency of perhaps a few hundred $\mathrm{kHz}$ (probably not impossible, though). Secondly, the rigid reference cavity will not have the fractional length stability necessary to meet the frequency stability requirements. Then as the stabilisation to the interferometer arms can give a gain of about 100 around $1 \mathrm{kHz}$, the need for prestabilisation is now $10^{-4} \mathrm{~Hz} / \mathrm{JHz}$, and our work on the prestabilisation experiment has been of two steps: firstly having very low noise servo-loop with very high gain at low freqencies $\left(10^{5}\right)$ and secondly designing very low noise reference cavities with seismic, thermal noises below $10^{-4} \mathrm{~Hz} / \mathrm{VHz}$.

A block diagram of the prestabilization frequency made by using the double PZT and EO loops, is shown in Figure 3. The laser is frequency locked on a rigid cavity using the Drever-Hall technique, by interrogating the cavity in reflection with sidebands obtained by phase modulation of the light. A Lightwave Electronics NPRO $300 \mathrm{~mW}$ Nd:YAG laser is used for the experiment: the system includes electronic damping of the relaxation oscillations; the ring geometry of the laser makes it less sensitive to reflections, and there is also thermal tuning of the laser frequency over roughly $10 \mathrm{GHz}$, so there will be no problems holding locks over long time periods.

The spectra shown on the figure 4 give the spectral density of the error signal fluctuations vs the frequency compared to the shot noise level, when the laser is locked. One can see that this error signal representing the electronic noise of the servo-loop is below the shot noise limit. So the laser will follow the stability of the reference cavity better than $10^{-4} \mathrm{~Hz} / \sqrt{\mathrm{Hz}}$. 


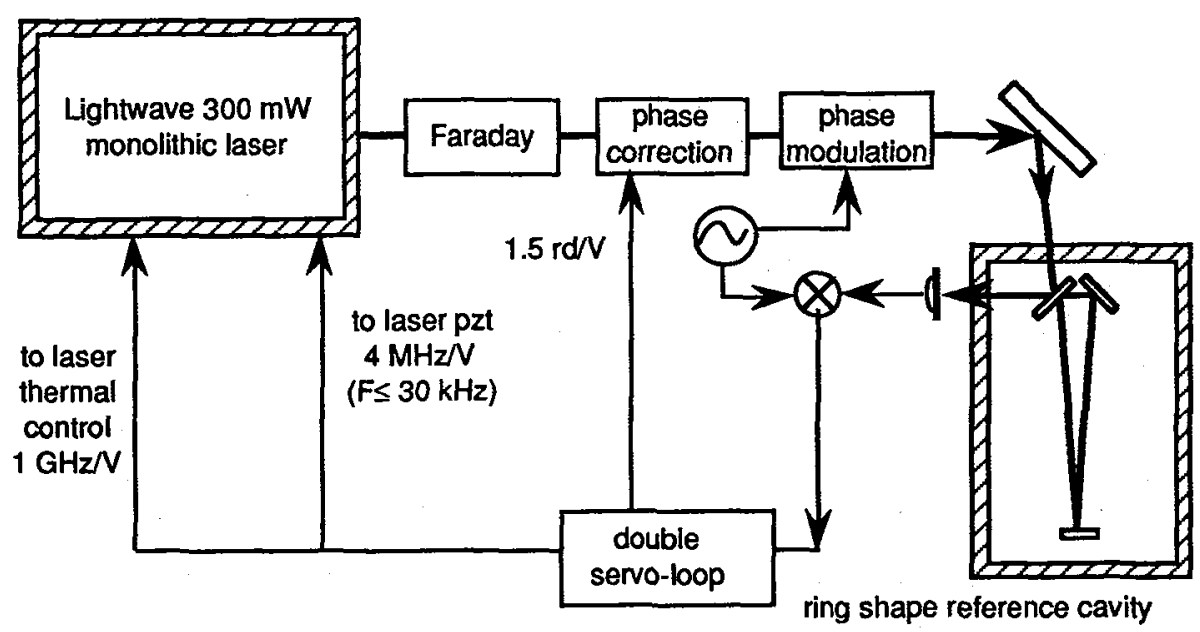

Figure 3: Block diagram of the frequency prestabilization on a rigid cavity

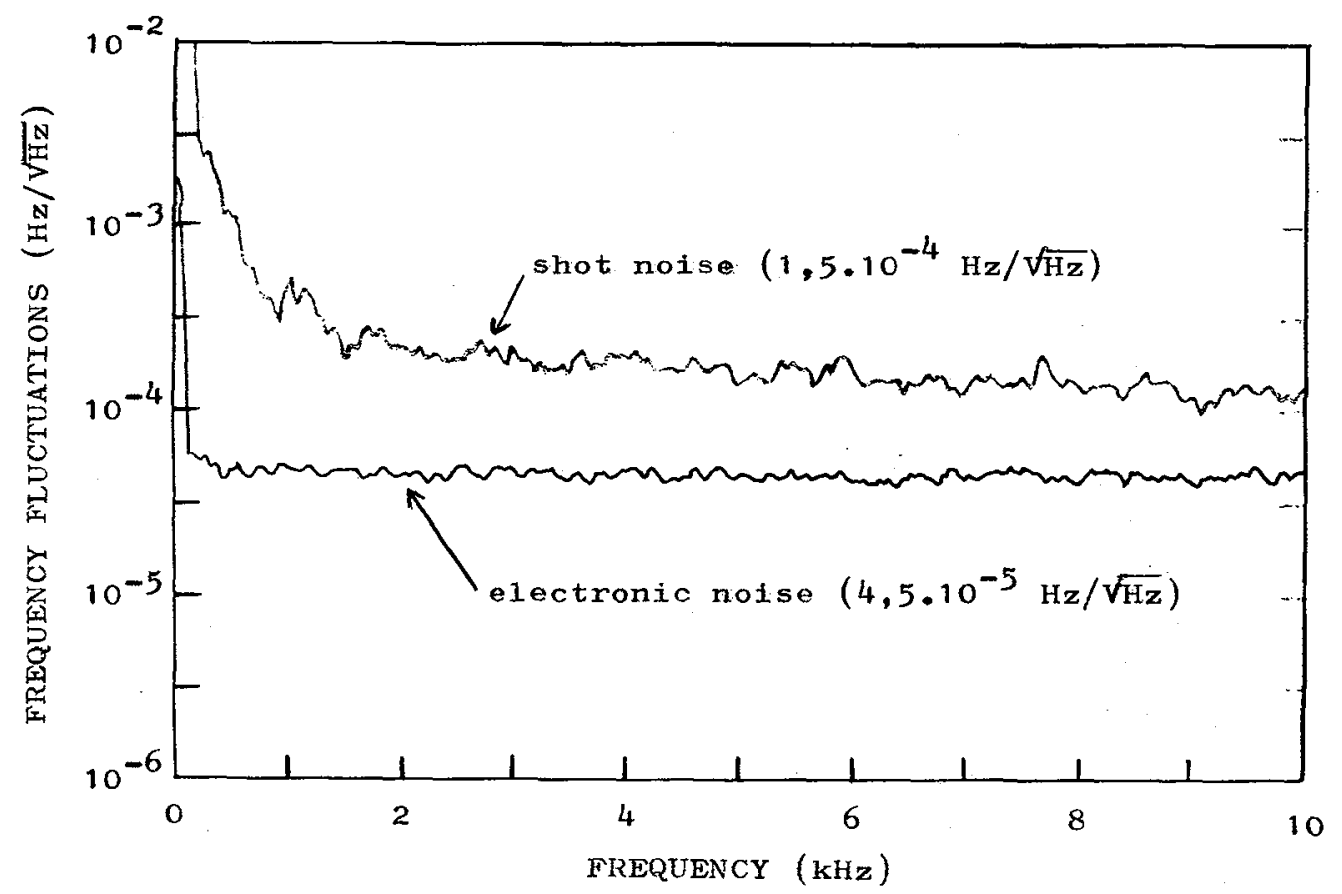

Figure 4 : Linear spectral density of the error signal fluctuations 


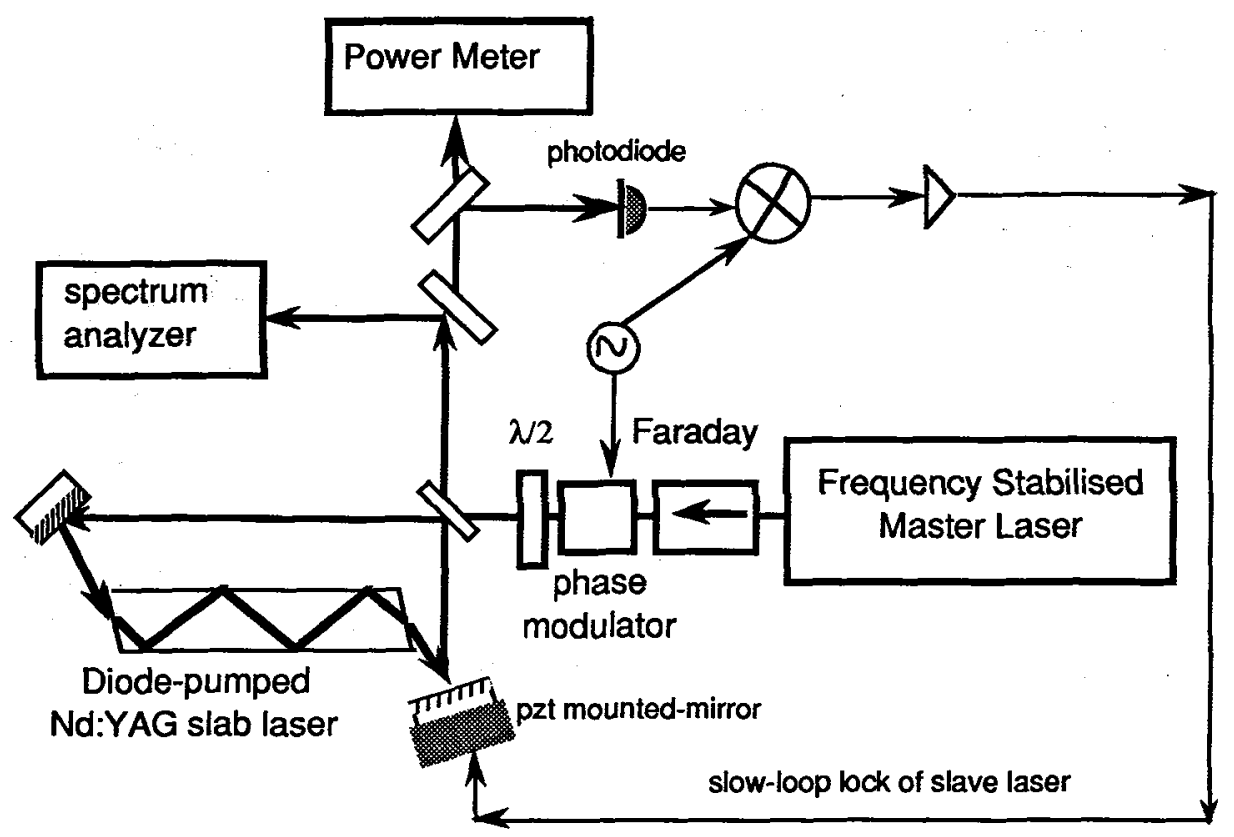

Figure 5 : Injection Locking of the ring shape slab laser

The figure 5 shows the injection locking set-up of the high power laser by the frequency stabilised master laser, using a $80 \mathrm{~mW}$ power of the master laser and getting a $700 \mathrm{kHz}$ injection locking range. The slow-loop is used to hold the two laser frequencies within the injection locking when there are strong mechanical or acoustical perturbations.

3. Amplitude noise: the residual amplitude fluctuations of the laser can be converted into phase shifts at the output, for any asymmetry between the arms. The main effects originate from the radiation pressure on the mirrors and an asymmetry of $10^{-3}$ gives rise to a requirement in spectral density of the amplitude fluctuations of less than $10^{-6} / \mathrm{NHz}$ at a frequency of $1 \mathrm{kHz}$. This requirement is relatively easy to fulfill for a monolithic laser such as the Lightwave laser, at low frequencies the laser noise is roughly $\delta \widetilde{P} / P \approx 2.5 \times 10^{-6}(10 \mathrm{~Hz} / f)^{1 / 2},(\operatorname{good}$ from $10 \mathrm{~Hz}-10 \mathrm{kHz})$. The relaxation oscillation peak inherent to all solid state laser is at $470 \mathrm{kHz}$, but this has been suppressed easily by a damping circuit included in the power supply. 
4. Beam pointing noise: again for an asymmetry between the arms, any beam pointing fluctuations of the laser induces phase shifts at the output; for a beam size of 2 $\mathrm{cm}$ in waist this beam pointing spectral density has to be less than $10^{-9} \mathrm{rd} / \sqrt{\mathrm{Hz}}$. Using a quadrant photodiode, we have measured the position noise of our stabilized laser and it corresponds to either $\tilde{\delta x} \approx 10^{-11} \mathrm{~m} / \sqrt{\mathrm{Hz}}$ or $\tilde{\delta \alpha} \approx 10^{-11} \mathrm{rad} / \sqrt{\mathrm{Hz}}$ at frequencies around $1 \mathrm{kHz}$ (lower frequencies are limited by the stability of the photodiode) and these numbers are lower than the requirements listed earlier.

The requirements listed above for the laser source has to be fulfilled simultaneously by the laser, taking into account the fact that each noise source influences the other. Besides all these required specifications for the laser, it has to be extremely reliable and operate continously 24 hours a day. The use of diode-pumped Nd:YAG laser in Virgo will allow us to benefit of the long lifetime of the diodes ( $>10000$ hours) and the multiple diode pumping scheme will prevent any stop of the YAG laser in case of failure of one of the diodes.

\section{Conclusion}

We have described the laser characteristics needed for the Virgo project of interferometric detection of gravitational waves in terms of power, stabilities in frequency, intensity and beam jitterings; though they are all at the limits of the today stateof art, we have described here the solutions we adopted to overcome the difficulties and showed the first results leading us into the right direction.

\section{References}

[1] J.R.Taylor and J.M.Weisberg, Astrophysical Journal, 253, 908-920 (1982)

[2] M.E.Gertsenshtein and V.I.Pustovoit, Soviet Physics JETP,16, 433 (1963)

[3] R.Weiss, Quart.Progr.Rep.Res.Lab.MIT 105, 54 (1972)

[4] G.E.Moss, L.R.Miller, R.L.Forward, Applied Optics, 10, 2495 (1971)

[5] C.N.Man and A.Brillet, 1984 Cleo Proceed. paper TuB36

[6] C.N.Man, A.Brillet and D.Shoemaker, Gravitational wave data analysis Natoworkshop, Cardiff July 1987

[7] C.N.Man et al. Physics Letters A, 148,8 (1990)

[8] D.H.Shoemaker et al. Optics Letters, 14, 609 (1989)

[9] C.Bradaschia et al., Phys.Lett.A, 163, 1520 (1992)

[10] P.Tourrenc, N.Deruelle, Ann Phys.Fr. 10, 241-252 (1985) 